\title{
Efficient soliton self-frequency shift in hydrogen-filled hollow-core fiber
}

\author{
Yi-Hao Chen, ${ }^{1,{ }^{*}}$ and Pavel Sidorenko ${ }^{1}$ and Enrique \\ ANTONIO-LOPEZ ${ }^{2}$ AND ROdRIGo AMEZCUA-CORREA ${ }^{2}$ AND Frank \\ WISE ${ }^{1}$ \\ ${ }^{1}$ School of Applied and Engineering Physics, Cornell University, Ithaca NY 14853, USA \\ ${ }^{2}$ University of Central Florida, CREOL, The College of Optics and Photonics, Orlando, Florida 32816, \\ USA \\ *yc2368@cornell.edu
}

\begin{abstract}
We report a study of soliton self-frequency shifting in hydrogen-filled hollow-core fiber. The combination of hydrogen and short 40 -fs input pulses underlies clean and efficient generation of Raman solitons between 1080 and $1600 \mathrm{~nm}$. With 240-nJ input pulses, the Raman soliton energy ranges from 110 to $20 \mathrm{~nJ}$ over that wavelength range, and the pulse duration is approximately $45 \mathrm{fs}$. In particular, 70-nJ and 42 -fs pulses are generated at $1300 \mathrm{~nm}$. Numerical simulations agree reasonably well with experiments and predict that microjoule-energy tunable pulses should be possible with higher-energy input pulses.

(c) 2021 Optica Publishing Group. One print or electronic copy may be made for personal use only. Systematic reproduction and distribution, duplication of any material in this paper for a fee or for commercial purposes, or modifications of the content of this paper are prohibited.
\end{abstract}

Recently, hollow-core fibers have attracted a lot of attention due to the abundant physics that is possible when pulses inside a fiber interact with various gases. With inert gases, only the electronic Kerr response takes place, and such fibers have been used in applications such as ultraviolet generation [1], photoionization-induced blue-shift [2], pulse compression, and supercontinuum generation [3-5]. In molecular gases, Raman effects are important. In contrast to solid-core silica fibers, Raman scattering in molecular gases has a long dephasing time ( $\sim 100 \mathrm{ps}$ depending on the gas pressure) [6]; this underlies interesting phenomena that are unique to gases. Raman-enhanced Kerr nonlinearity [7] and solid-state physics in a gas-induced temporal crystal [8], just to name a few effects, have been demonstrated. Loranger et al. showed that 40-fs pulses at $1800 \mathrm{~nm}$ can be generated by launching $300 \mathrm{fs}$ pulses into a hydrogen-filled hollow-core fiber [9]. Initial observations of the soliton self-frequency shift (SSFS) in gas-filled hollow-core fibers reported small spectral shifts of tens of nanometers [10-12]. Stimulated Raman scattering plays a role in several recent studies, especially those of continuum generation and soliton self-compression $[13,14]$. The interplay of Raman and multimode effects has led to the recent observation of multidimensional solitary states [15]. Despite the importance of Raman effects, there is no systematic study of SSFS in hollow-core fibers to the best of our knowledge.

SSFS has long been a good candidate for wavelength-tunable fiber sources [16-18]. In particular, nonlinear microscopy, such as three-photon imaging, requires high peak power at 1300 and $1700 \mathrm{~nm}$ to overcome the depth limit of two-photon imaging $[19,20]$. SSFS occurs when intrapulse Raman scattering continuously transfers energy from the high-frequency part of a soliton to the low-frequency part in an anomalous-dispersion medium [21,22]. The Raman soliton thus gradually shifts to the red as it propagates. Highly nonlinear photonic crystal fibers [23] and fibers made with different materials, e.g., tellurite glasses [17], were used to produce Raman solitons in various wavelength ranges but with limited energies. The use of large-mode-area (LMA) silica fiber allowed the generation of pulses tunable from 1580 to $2130 \mathrm{~nm}$, with up to 45-nJ pulse energy and 70-fs pulse duration [18]. With a solid-core anti-resonant photonic crystal 
fiber, 95-nJ and 85-fs pulses at $1800 \mathrm{~nm}$ were generated [24]. The process of soliton self-mode conversion (SSMC) exploits higher-order modes of multimode fiber to achieve anomalous dispersion and large mode area at wavelengths below $1300 \mathrm{~nm}$ [25]. SSMC has yielded 80-nJ and 74-fs pulses at $1300 \mathrm{~nm}$. Although they have impressive peak power, further scaling of the pulse energy in solid-glass fibers appears to be difficult.

In contrast to solid-core fibers, nonlinearity of gases is low and high-energy solitons can propagate through a gas-filled fiber with a low soliton number. In addition, the pressure-tunable dispersion and broad transmission bands [3] of anti-resonant hollow-core fibers (AR-HCFs) make this platform extremely attractive for high-energy SSFS.

In this letter, we demonstrate SSFS in a hydrogen-filled AR-HCF. Thanks to the use of hydrogen and short input pulses, efficient and clean SSFS occurs. Continuous tuning of the wavelength between 1080 and $1600 \mathrm{~nm}$ is observed. Pulse energies in the range of 20 to $110 \mathrm{~nJ}$ and durations below $50 \mathrm{fs}$ are obtained over this spectral range. Numerical simulations account well for the experimental results and predict scaling of the process to microjoule energies with stronger pump pulses.

To generate a clean Raman soliton with high efficiency, both the soliton number of the launched pulse and the number of participating Raman transitions should be as small as possible. Several studies have obtained wavelength-tunable sources by extracting the reddest lobe from a supercontinuum $[13,14,26]$; however, they failed to meet the above conditions and had relatively low efficiency. For example, with $\mathrm{N}_{2}$, Carpeggiani et al. demonstrated $57 \mathrm{fs}$ pulses with $8 \%$ efficiency in the 1450-1650-nm spectral window. The strong launched pulse evolves as a high-order soliton with a soliton number $N$ given by

$$
N^{2}=\frac{\gamma P_{0} T_{0}^{2}}{\left|\beta_{2}\right|} \approx \frac{\gamma E_{0} T_{0}}{\left|\beta_{2}\right|}
$$

where $\gamma$ is the nonlinear coefficient, $P_{0}$ is the peak power, $T_{0}$ is the pulse duration, $E_{0} \approx P_{0} T_{0}$ is the pulse energy, and $\beta_{2}$ is the dispersion. Perturbations from higher-order dispersion, selfsteepening, etc., cause the field to undergo fission into $N$ constituent fundamental solitons. A pulse of a smaller soliton number breaks into fewer fundamental solitons, so the reddest Raman soliton takes up more energy. Soliton number is directly proportional to the pulse duration, so a shorter input pulse will lead to a more efficient SSFS process. In addition, pulse bandwidth needs to be large enough to support the SSFS. Recently, a new fiber amplification regime that allows the generation of pulses with spectra well beyond the gain-narrowing limit was demonstrated [27,28]. With LMA Yb-doped fiber, such a gain-managed amplifier can deliver 1- $\mu \mathrm{J}$ and $40 \mathrm{fs}$ pulses. The short pulse duration makes it an ideal simple source for investigation of SSFS with small soliton number. We choose $\mathrm{H}_{2}$ over other gases to minimize the number of Raman transitions that can occur. Because it has a large energy difference between energy states, most of the population stays in the lowest possible states at room temperature. Only two rotational transitions, $\mathrm{S}(0)$ with para- $\mathrm{H}_{2}(\Delta v=0, J=0 \rightarrow 2)$ and $\mathrm{S}(1)$ with ortho- $\mathrm{H}_{2}(\Delta v=0, J=1 \rightarrow 3)$, and one vibrational Raman transition, $\mathrm{Q}(0)(v=0 \rightarrow 1, \Delta J=0)$, play important roles. Because the ratio of ortho- $\mathrm{H}_{2}$ to para- $\mathrm{H}_{2}$ populations is 3:1 at room temperature, $\mathrm{S}(1)$ dominates over $\mathrm{S}(0)$. Despite the larger Raman gain of the vibrational Raman transitions than the rotational ones, self-seeded SSFS of a sub-50-fs broadband pulse dominates over the noise-seeded discrete vibrational Raman transitions. As mentioned above, several studies have investigated Raman-shifting in nitrogen-filled fibers. With $\mathrm{N}_{2}$, multiple rotational Raman transitions come into play, which increases the pulse duration and reduces the efficiency of conversion to a single wavelength (see the discussion of nitrogen-filled AR-HCF in Supplement 1).

The experimental setup is depicted in Fig. 1(a). A gain-managed amplifier supplies 400-nJ and 32-fs pulses [Fig. 1(b) and 1(c)]. The linearly-polarized pulses are coupled, with $60 \%$ efficiency, into a 2-m AR-HCF with a 30- $\mu \mathrm{m}$ core diameter and 300-nm wall thickness of its 
tubes [Fig. 1(d)]. By paying more attention to the coupling optics, coupling up to $90 \%$ should be achievable. The fiber is designed to have less than $0.3-\mathrm{dB} / \mathrm{m}$ loss between 800 and $1700 \mathrm{~nm}$ [Fig. 1(e)] [29]. It maintains anomalous dispersion at wavelengths above $1 \mu \mathrm{m}$ for hydrogen pressures up to 100 bar [Fig 1(f)] [30]. Propagation in the fundamental transverse mode of the fiber was confirmed (see Fig. S2 in Supplement 1).

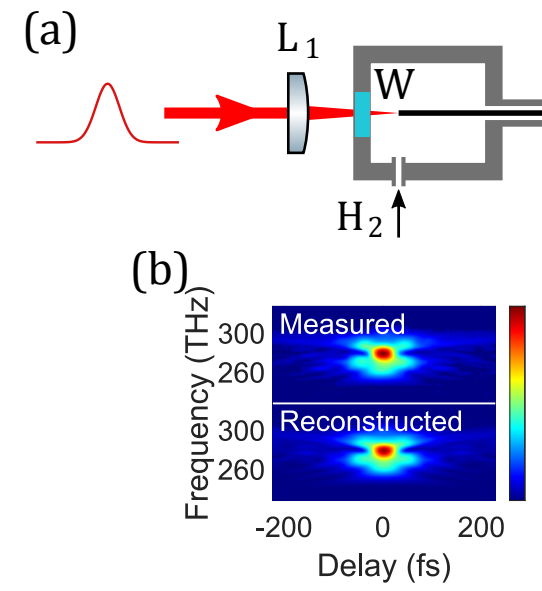

(d)

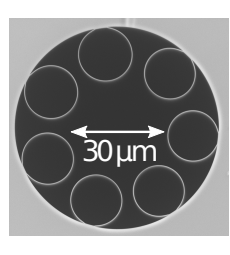

(e)

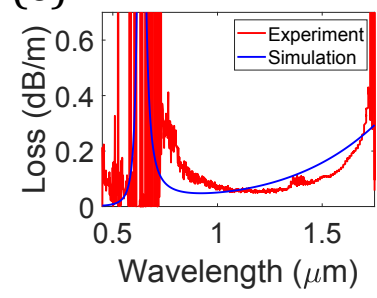

(c)

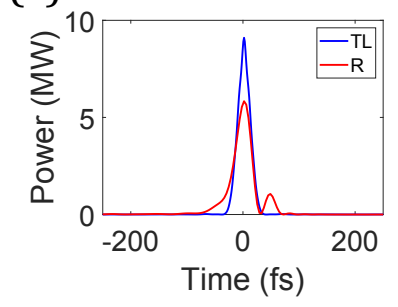

(f)

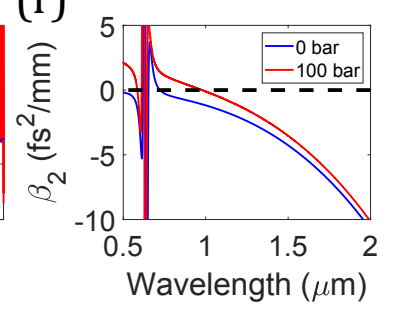

Fig. 1. (a) Schematic of the gas system. Two gas cells are connected with a stainlesssteel tube where the AR-HCF lies. $L_{1}$ and $L_{2}$ are focusing and collimating lenses with 50-mm focal length; LPF is a long-pass filter for selecting the Raman soliton; $W$ is a sapphire window. (b) Measured and reconstructed FROG traces of the input pulse. (c) Temporal profiles of the retrieved (R) and transform-limited (TL) input pulses. Measured (d) cross section and (e) loss curve of the seven-tube AR-HCF. (f) Calculated dispersion curves of the fiber with vacuum and 100-bar hydrogen pressure. The zero-dispersion wavelengths are $720 \mathrm{~nm}$ and $980 \mathrm{~nm}$, respectively.

The experimental results are summarized in Fig. 2. The measured output spectra [Fig. 2(a)] exhibit a red-shifting peak on the long-wavelength side of the spectra, which contributes entirely to a single Raman soliton, as expected owing to the excitation of primarily a single rotational Raman transition. The frequency shift increases roughly linearly with $\mathrm{H}_{2}$ pressure up to about $90 \mathrm{bar}$, where it seems to saturate. At 98.2 bar, we observe a soliton at $1600 \mathrm{~nm}$. After isolating the Raman soliton with a long-pass spectral filter, we measure its pulse duration. The pulse duration measured by frequency-resolved optical gating (FROG) is around $45 \mathrm{fs}$ for all pressures in the range investigated [Fig. 2(b)]. The pulse energy varies from above $100 \mathrm{~nJ}$ for small frequency shifts to $20 \mathrm{~nJ}$ at the largest shift. The peak power of the Raman soliton thus exceeds $1 \mathrm{MW}$ for pressures (wavelengths) up to 50-bar (1430 nm). With 40-bar $\mathrm{H}_{2}$ pressure, we obtain $70-\mathrm{nJ}$ and 42 -fs pulses at $1300 \mathrm{~nm}$, for a peak power of nearly $2 \mathrm{MW}$ at this biologically-important wavelength.

In addition to the Raman soliton, we observe dispersive waves in the visible spectral region for gas pressures between 50 and 80 bar. The sharp dispersion slope near the resonance enables 
phase-matched dispersive-wave generation despite the high confinement loss [31] (see the discussion of dispersive waves in Supplement 1).

(a)

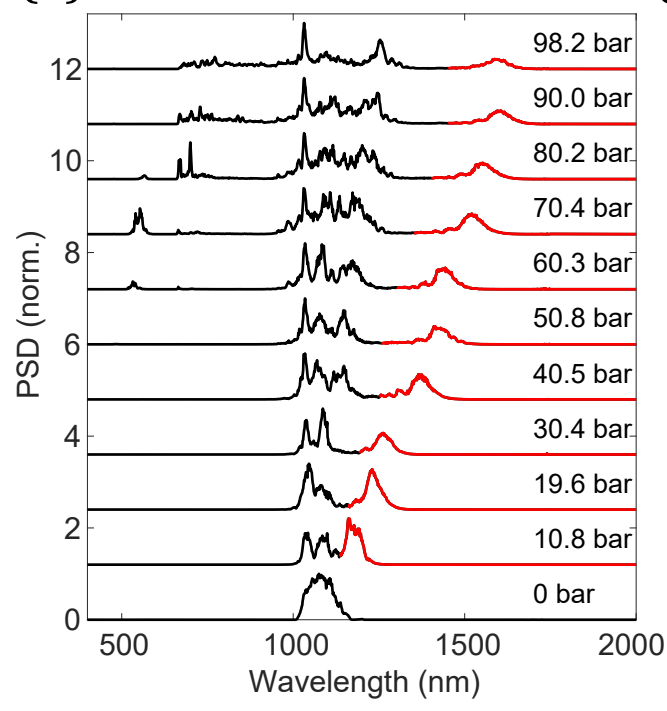

(b)

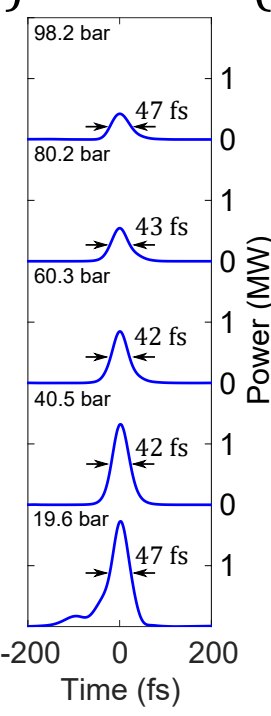

(c)

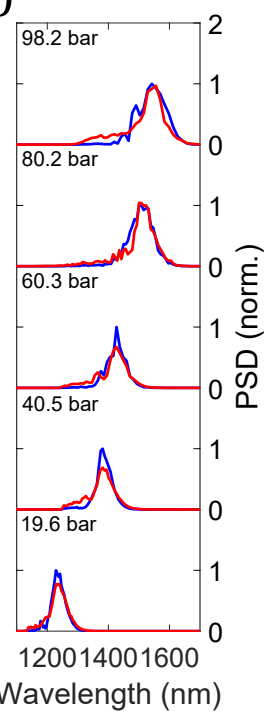

Fig. 2. (a) Output spectra (red curve corresponds to the band transmitted by the long-pass filer). PSD: power spectral density. (b) Raman pulses measured with FROG at indicated pressures. (c) Spectra retrieved with FROG (blue) and measured with an optical spectrum analyzer (red).

To obtain insight into the experimental results, we numerically simulated the process with a unidirectional pulse propagation equation that includes the electronic and Raman nonlinearities of $\mathrm{H}_{2}$ (details are in Supplement 1),

$$
\begin{aligned}
& \partial_{z} A(z, \Omega)=i\left[\beta(\omega)-\left(\beta_{0}+\beta_{1} \Omega\right)\right] A(z, \Omega)+ \\
& \frac{i \omega}{4} Q_{1111}^{R}\left(\frac{3 \epsilon_{0} \chi_{\text {electronic }}^{(3)}}{4} \mathfrak{F}\left[|A|^{2} A\right]+\mathfrak{F}\left[A\left[R(t) *\left(|A|^{2}\right)\right]\right]\right),
\end{aligned}
$$

where $z$ is the propagation distance, $\omega$ and $\Omega=\omega-\omega_{0}$ are the angular frequency and the frequency offset from the center of the frequency window, $A(z, \Omega)$ is the fundamental-mode electric field, $\beta(\omega)$ is the propagation constant including the effect of the gas, $\beta_{0}$ and $\beta_{1}$ are the free parameters of the model where $\beta_{1}=1 / v_{g}$ represents the inverse moving speed of the reference frame in simulations. We apply the simplified model proposed by Bache et al. to compute both the loss and the propagation constant of the propagating mode [30]. $Q_{1111}^{R}=\frac{4}{\epsilon_{0}^{2} n_{\mathrm{eff}}^{2}{ }^{2}} \frac{1}{A_{\mathrm{eff}}}$ in which $n_{\mathrm{eff}}=\frac{\beta(\omega)}{k_{0}}$ and $A_{\text {eff }}$ represent the effective refractive index and the effective area of the mode. $\chi_{\text {electronic }}^{(3)}$ is the Kerr nonlinear coefficient. The total Raman response includes vibrational and rotational parts, $R(t)=R^{\mathrm{rot}}(t)+R^{\mathrm{vib}}(t)$. The vibrational response

$$
\begin{aligned}
& R^{\mathrm{vib}}=N_{g} \frac{1}{4 \mu} \mathrm{e}^{-\gamma_{2}^{\mathrm{vib}} t} \times \\
& \sum_{J}(2 J+1) \rho_{J}^{(0)} \frac{\left(\frac{\mathrm{d} \alpha}{\mathrm{dQ}}\right)_{0}^{2}+\frac{4}{45} \frac{J(J+1)}{(2 J-1)(2 J+3)}\left(\frac{\mathrm{d}(\Delta \alpha)}{\mathrm{dQ}}\right)_{0}^{2}}{\omega_{1 J, 0 J}} \sin \left(\omega_{1 J, 0 J} t\right)
\end{aligned}
$$


and the rotational response

$$
\begin{aligned}
R^{\mathrm{rot}} & =N_{g} \frac{1}{15 \hbar}(\Delta \alpha)^{2} \mathrm{e}^{-\gamma_{2}^{\mathrm{rot}} \times} \times \\
& \sum_{J}\left(\rho_{J}^{(0)}-\rho_{J+2}^{(0)}\right) \frac{(J+2)(J+1)}{2 J+3} \sin \left(\omega_{0 J+2,0 J} t\right),
\end{aligned}
$$

are both summed over the rotational quantum number $J . N_{g}$ is the number density, $\mu$ is the reduced mass of the gas molecule, $\gamma_{2}$ is the dephasing rate, $\rho_{J}^{(0)}$ is the Boltzmann-distributed population of the energy state $(v=0, J)$ without the perturbed electric field, $\left(\frac{\mathrm{d} \alpha}{\mathrm{dQ}}\right)_{0}$ is the derivative of the gas mean polarizability with respect to the normal coordinate of the molecule at equilibrium, $\Delta \alpha$ is the polarizability anisotropy, and $\omega_{v_{1} J_{1}, \nu_{2} J_{2}}=\Delta E_{v_{1} J_{1}, v_{2} J_{2}} / \hbar$ represents the energy difference between two states.

Figs. 3 and 4 summarize the simulation results. The Raman soliton shifts more with increasing gas pressure [Fig. 3(a)]. Although multiple Raman transitions are included, SSFS generates clean red-shifted solitons. We also observe dispersive waves around $550 \mathrm{~nm}$ and residual energy around $1 \mu \mathrm{m}$. These spectral features are all consistent with the experimental results. The simulated pulse durations are between 30 to $41 \mathrm{fs}$, somewhat shorter than observed experimentally. This results from the uncompensated dispersion from lenses, the sapphire window, the long-pass filter, and the polarizing beam splitter cube. As an example, the spectral evolution of the pulse at 60-bar gas pressure is shown in Fig. 3(c). At the beginning, several fundamental solitons in a high-order soliton experience different levels of rotational Raman scattering. Eventually the reddest one temporally separates from the rest of the solitons and initiates the smooth spectral red-shift as in Fig. 3(c). This reddest Raman soliton is clear in the temporal evolution [Fig. 3(d)]. Both the simulated pulse energy and the spectral shift exhibit the same trends as the experiments. The soliton number goes from 1.5 to 7.5 when the gas pressure increases, which accounts for the decreasing efficiency with higher pressure (beyond the quantum efficiency of the Raman scattering). However, the simulations predict Raman solitons with energies about $30 \mathrm{~nJ}$ higher than observed [Fig. 4(a)], along with soliton wavelengths up to $1800 \mathrm{~nm}$ [Fig. 4(b)]. The simulated spectra also exhibit broader and more-structured dispersive-wave peaks than the experiments. These discrepancies, while not major, are puzzling. We have not found reasonable combinations of parameters that can significantly reduce them. We tentatively attribute them to discrepancies between the actual fiber parameters and the simplified model of fiber propagation constants and loss, which are evident in Fig. 1(e).

The experimental results presented here are limited by the available input pulse energy. If higher-energy pulses are available, significant increases in the performance should be possible. To assess this, we performed simulations with $2-\mu \mathrm{J}$ and 35 -fs pulses launched into the same fiber considered above. Such a pulse could be obtained by compression of the output of a standard chirped-pulse amplifier, or by future scaling of the gain-managed concept. The higher pulse energy allows the gas pressure to be reduced. Since the dispersion of a gas-filled hollow-core fiber includes anomalous waveguide dispersion and normal gas dispersion, with the low gas pressure, the dispersion is more anomalous across the spectral range of interest. This, along with weaker nonlinearity, reduces the soliton number. Because generation of dispersive waves is not phase-matched at the lower pressures, no dispersive waves are observed in the output spectra. As a result, the efficiency of Raman-soliton production improves. Wavelengths as long as $1700 \mathrm{~nm}$ are generated, with 30-60\% efficiency (Fig. 5). Pulse energies above $1-\mu \mathrm{J}$ and peak powers around 30-MW are predicted. Ionization of the $\mathrm{H}_{2}$ should not be a concern at these peak powers; however, it could become significant for scaling to even higher pulse energies (see the discussion of ionization in Supplement 1).

In conclusion, we have theoretically and experimentally studied SSFS in hydrogen-filled AR-HCF. With short 1080-nm input pulses, wavelength tunability from 1080 to $1600 \mathrm{~nm}$ and 
(a)

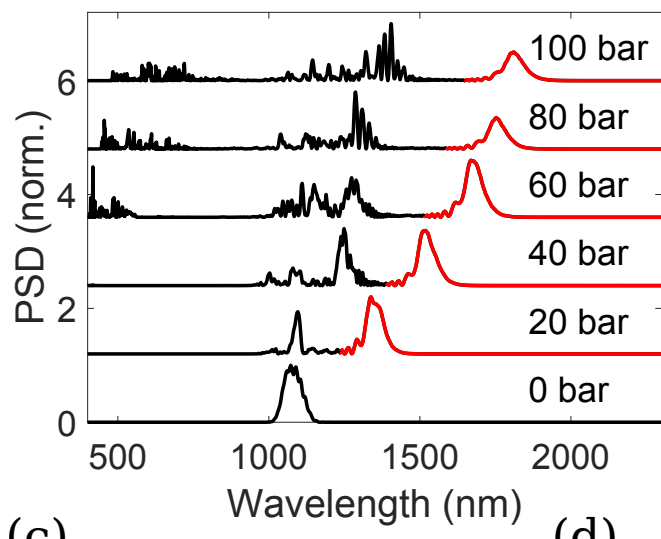

(b)

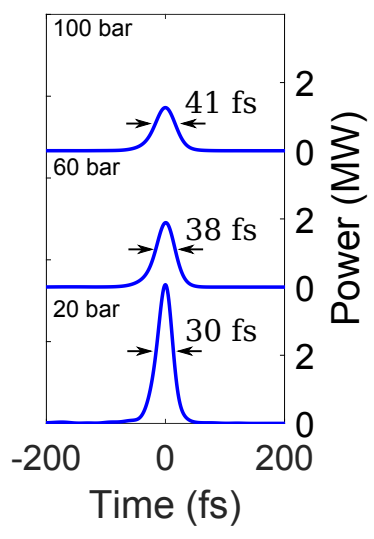

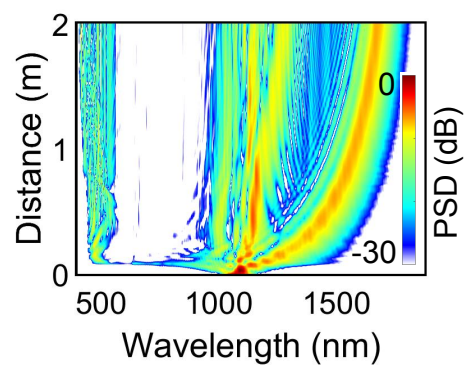

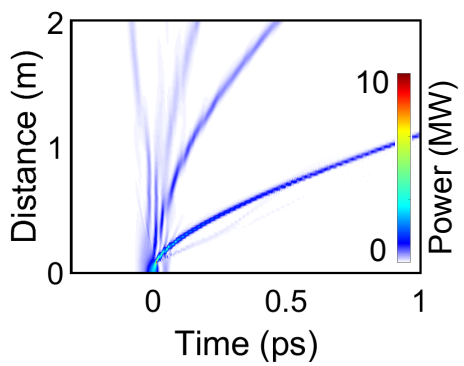

Fig. 3. Simulated (a) output spectra and (b) Raman pulses at the indicated $\mathrm{H}_{2}$ pressures. Simulated (c) spectral and (d) temporal evolution at 60-bar pressure.

(a)

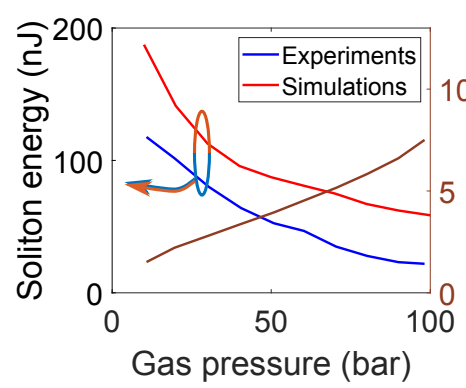

(b)

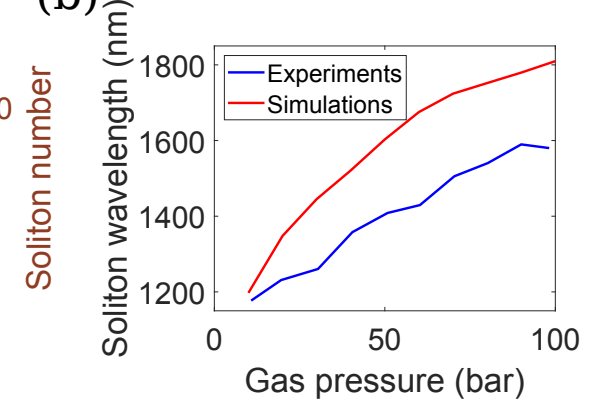

Fig. 4. (a) Raman soliton energy and soliton number of the input pulse and (b) Raman soliton wavelengths versus gas pressure for both the experiments and the simulations. The launched pulse energy is $240 \mathrm{~nJ}$.

consistent 45 -fs pulse duration are achieved. The generated Raman solitons reach peak powers around $1 \mathrm{MW}$, and scaling to an order of magnitude higher peak power should be possible.

Funding. Office of Naval Research (N00014-19-1-2592), National Institutes of Health (EB002019), Army Research Office (65016372), and Air Force Research Laboratory (FA86511820019).

Acknowledgments. The authors are grateful to Francesco Tani for advice on building the gas cells and David Novoa for advice on the simulation code.

Disclosures. The authors declare no conflicts of interest. 
(a)

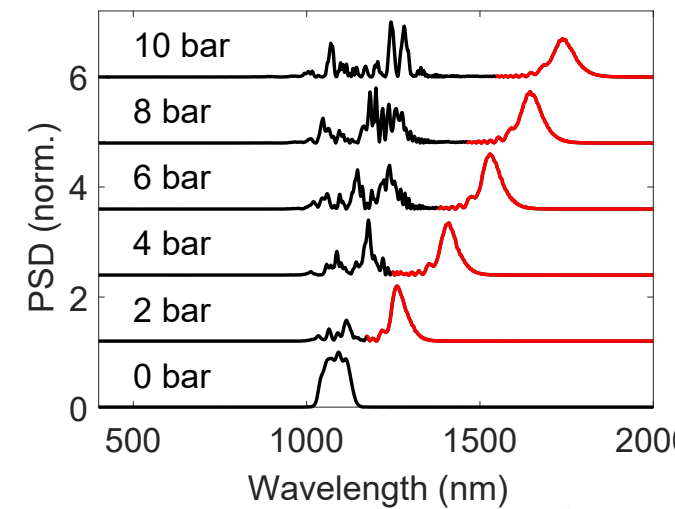

(c)

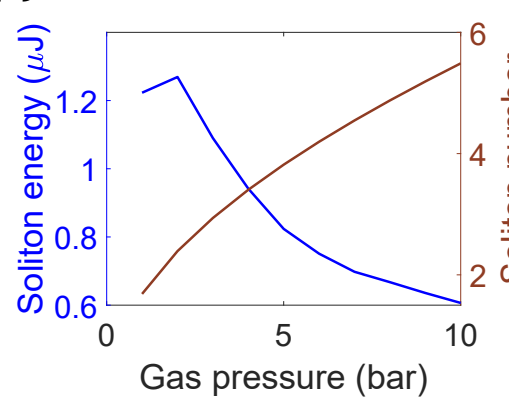

(b)

(d)

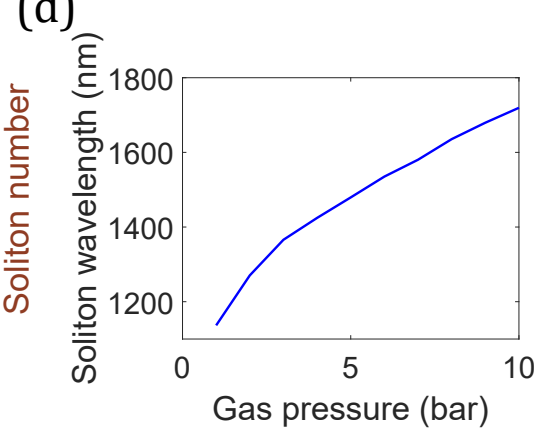

Fig. 5. Simulations with $2-\mu \mathrm{J}$ and $35-$ fs input pulse. (a) Output spectra and (b) Raman pulses at the indicated $\mathrm{H}_{2}$ pressures. (c) Raman soliton energy, soliton numbers of the pump pulses, and (d) Raman soliton wavelengths at different gas pressures.

Data availability. Data underlying the results presented in this paper are not publicly available at this time but may be obtained from the authors upon reasonable request.

Supplemental document. See Supplement 1 for supporting content. 


\title{
Efficient soliton self-frequency shift in hydrogen-filled hollow-core fiber: supplemental document
}

\begin{abstract}
The supplementary material in this document is organized as follows: In the manuscript, pulse propagation inside a hollow-core fiber is modeled with a unidirectional pulse propagation equation (UPPE) that includes electronic and (vibrational and rotational) Raman responses. The derivation of the equation is presented in Section 1. Section 2 describes simulations of SSFS in a fiber filled with $\mathrm{N}_{2}$. These demonstrate SSFS with lower efficiency due to many Raman transitions that play out equally. Section 3 presents measured output spatial profiles of the filtered Raman solitons generated in the hydrogen-filled fiber, which confirm single-mode propagation. Section 4 discusses, in detail, the observed resonance-induced dispersive-wave generation in Fig. 2 and Fig. 3. Section 5 is a brief discussion of the possible role of photoionization in future work on scaling results presented here to higher pulse energies.

(c) 2021 Optica Publishing Group. One print or electronic copy may be made for personal use only. Systematic reproduction and distribution, duplication of any material in this paper for a fee or for commercial purposes, or modifications of the content of this paper are prohibited.
\end{abstract}

\section{UPPE with delayed Raman response of a gas}

\subsection{UPPE}

The convention of the field follows the traditional generalized nonlinear Schrödinger equation (GNLSE) [32]:

$$
\begin{aligned}
\overrightarrow{\mathbb{E}}(\vec{x}, t) & =\frac{1}{2}[\overrightarrow{\mathcal{E}}(\vec{x}, t)+\text { c.c. }] \\
& =\int \mathrm{d} \omega \frac{1}{2}\left\{\frac{\vec{F}(x, y, \omega)}{N(\omega)} A(z, \omega) \mathrm{e}^{i[\beta(\omega) z-\omega t]}+\text { c.c. }\right\} \\
& =\frac{1}{2}\left\{\frac{\vec{F}(x, y)}{N}\left[A(z, t) \mathrm{e}^{i\left(\beta_{0} z-\omega_{0} t\right)}\right]+\text { c.c. }\right\}, \text { assume } \vec{F}(x, y, \omega)=\vec{F}(x, y) \\
& =\frac{1}{2}\left\{\frac{\vec{F}(x, y)}{\sqrt{\frac{\epsilon_{0} n_{\text {eff }}}{2}}}\left[A(z, t) \mathrm{e}^{i\left(\beta_{0} z-\omega_{0} t\right)}\right]+\text { c.c. }\right\},
\end{aligned}
$$

where $\overrightarrow{\mathcal{E}}(\vec{x}, t)$ is the analytic signal of the real-valued electric field $\overrightarrow{\mathbb{E}}(\vec{x}, t)$, "c.c." stands for complex conjugate, $\vec{F}(x, y)$ is the normalized spatial mode profile of the fundamental mode with the normalization condition, $\int|\vec{F}|^{2} \mathrm{~d}^{2} x=1$, and is assumed to be independent of frequency. $\overrightarrow{\mathbb{E}}(\vec{x}, t)$ has the unit of $\mathrm{V} / \mathrm{m} . A(z, t)$ represents the field and is normalized to have the unit of $\sqrt{\mathrm{W}}$ with the normalization constant $N=\sqrt{\frac{\epsilon_{0} n_{\mathrm{eff}} c}{2}} . \beta(\omega)=n_{\mathrm{eff}}(\omega) k_{0}$ is the propagation constant. $\beta_{0}$ and $\omega_{0}$ are two free parameters, usually chosen as the propagation constant and the angular frequency at the center frequency of a pulse. $A$ has the following time-frequency relation,

$$
A(z, t)=\int \mathrm{d} \omega A(z, \omega) \mathrm{e}^{i\left[\left(\beta(\omega)-\beta_{0}\right) z-\left(\omega-\omega_{0}\right) t\right]}
$$


The scalar-field UPPE is

$$
\partial_{z} A(z, \Omega)=i\left[\beta(\omega)-\left(\beta_{0}+\beta_{1} \Omega\right)\right] A(z, \Omega)+\frac{i \omega}{4 N^{2}} P(z, \Omega),
$$

where $\beta_{1}$ is the inverse group velocity of the moving frame, the analytic signal of the nonlinear polarization $\overrightarrow{\mathcal{P}}(\vec{x}, t)=\frac{\vec{F}(x, y)}{N} P(z, t) \mathrm{e}^{i\left(\beta_{0} z-\omega_{0} t\right)}$ and $\Omega=\omega-\omega_{0}$. By expanding $P(z, \Omega)$ into Kerr and Raman terms for the linearly-polarized field, it becomes

$$
\begin{aligned}
\partial_{z} A(z, \Omega)= & i\left[\beta(\omega)-\left(\beta_{0}+\beta_{1} \Omega\right)\right] A(z, \Omega)+ \\
& \frac{i \omega}{\epsilon_{0}^{2} n_{\text {eff }}^{2} c^{2} A_{\text {eff }}(\omega)}\left(\frac{3 \epsilon_{0} \chi_{\text {electronic }}^{(3)}}{4} \mathfrak{F}\left[|A|^{2} A\right]+\mathfrak{F}\left[A\left[R(t) *\left(|A|^{2}\right)\right]\right]\right),
\end{aligned}
$$

where $R(t)=R^{\mathrm{rot}}(t)+R^{\mathrm{vib}}(t)$, the total Raman response including rotational and vibrational parts. $\mathfrak{F}$ stands for the Fourier Transform and $*$ for the convolution operation [33].

\subsection{Rotational Raman}

We modified the model of the rotational Raman response introduced by Chen et al. [34] and Wahlstrand et al. [35] to obtain a delayed Raman response, $R(t)$, of the form commonly used for silica fibers. Their model has been applied in several studies; however, instead of calculating the analytical form of the Raman response, they fit the calculated molecular orientation $\left\langle\cos ^{2} \theta\right\rangle_{t}$ to the Raman response function modeled with a damped harmonic oscillator $R(t)=R_{0} \mathrm{e}^{-\gamma_{R} t} \sin \left(\omega_{R} t\right)$ where $R_{0}$ is the normalization constant such that $\int R(t) \mathrm{d} t=1[13-15,26,36]$. Here, we derive an analytical expression for the rotational Raman response and show that it can be implemented in the UPPE [Eq. (8)] directly. By doing so, long-time features of the long dephasing times of gases, such as the 2.1-ps revivals of coherence or molecular re-alignment in $\mathrm{N}_{2}[37,38]$ or the 100 -ps ringing of the coherence wave in $\mathrm{H}_{2}$, can be captured.

We start with the dielectric response of diatomic gas molecules

$$
\begin{aligned}
\epsilon & =\epsilon_{0}+N_{g}\langle\alpha\rangle_{t} \\
& =\epsilon_{0}+N_{g}\left(\Delta \alpha\left\langle\cos ^{2} \theta\right\rangle_{t}+\alpha^{\perp}\right) \\
& =\epsilon(t \rightarrow-\infty)+N_{g} \Delta \alpha\left(\left\langle\cos ^{2} \theta\right\rangle_{t}-\frac{1}{3}\right),
\end{aligned}
$$

where $N_{g}$ is the molecular number density, $\Delta \alpha=\alpha_{\|}-\alpha_{\perp}$ is the polarizability anisotropy. $\alpha_{\|}$ and $\alpha_{\perp}$ are molecular polarizabilities when the electric field is parallel and perpendicular to the molecule, respectively. And

$$
\epsilon(t \rightarrow-\infty)=\epsilon_{0}+N_{g}\left(\frac{\triangle \alpha}{3}+\alpha^{\perp}\right)
$$

To solve $\left\langle\cos ^{2} \theta\right\rangle_{t}$, the density-matrix approach is applied.

$$
\left\langle\cos ^{2} \theta\right\rangle_{t}=\operatorname{Tr}\left[\hat{\rho} \cos ^{2} \theta\right]
$$

Here $\cos ^{2} \theta$ is treated as an operator and has $\left(\cos ^{2} \theta\right)_{k l}=\left\langle k\left|\cos ^{2} \theta\right| l\right\rangle$. $\hat{\rho}$ is the density matrix. From the perturbation theory [39], $\hat{\rho}=\hat{\rho}^{(0)}+\hat{\rho}^{(1)}$ where

$$
\rho_{k l}^{(1)}=-\frac{i}{\hbar} \int_{-\infty}^{t} \mathrm{~d} \tau\left[H_{\text {int }}(\tau), \hat{\rho}^{(0)}\right]_{k l} \mathrm{e}^{\left(\gamma_{k l}+i \omega_{k l}\right)(\tau-t)}
$$


is the first-order correction to the density matrix induced by the perturbed Hamiltonian,

$$
\begin{aligned}
H_{\mathrm{int}} & =\left\langle-\int \overrightarrow{\mathbb{E}} \cdot \mathrm{d} \vec{\mu}\right\rangle_{t} \\
& =\left\langle-\sum_{i, j} \alpha_{i j} \int \mathbb{E}^{i} \cdot \mathrm{d} \mathbb{E}^{j}\right\rangle_{t}=-\left\langle\sum_{i, j} e^{i} \alpha_{i j} e^{j}\right\rangle_{t} \int|\overrightarrow{\mathbb{E}}| \cdot \mathrm{d}|\overrightarrow{\mathbb{E}}|=-\frac{1}{2}\left[\Delta \alpha\left(\cos ^{2} \theta\right)+\alpha^{\perp}\right]|\overrightarrow{\mathbb{E}}|^{2},
\end{aligned}
$$

where $\vec{\mu}=\overline{\bar{\alpha}} \cdot \overrightarrow{\mathbb{E}}$.

As for the zeroth-order density matrix $\rho_{J, M}^{(0)}$,

$$
\begin{aligned}
& \rho_{J, M}^{(0)}=\frac{g_{J} \mathrm{e}^{-\frac{E_{J}}{k_{B} T}}}{Z} \\
& Z=\sum_{J} g_{J}(2 J+1) \mathrm{e}^{-\frac{E_{J}}{k_{B} T}} \\
& E_{J}=B_{e} J(J+1)-D_{e} J^{2}(J+1)^{2},
\end{aligned}
$$

where $g_{J}$ is the nuclear-spin statistical factor, and $B_{e}$ and $D_{e}$ are constants for the rotational energy states.

$$
\begin{aligned}
{\left[H_{\text {int }}(\tau), \hat{\rho}^{(0)}\right]_{k l} } & =\left(\rho_{l}^{(0)}-\rho_{k}^{(0)}\right) H_{\text {int }, k l}, \quad \because \rho_{k l}^{(0)}=\rho_{k}^{(0)} \delta_{k l} \text { when there's no external field } \\
& =\left(\rho_{l}^{(0)}-\rho_{k}^{(0)}\right) \frac{1}{2}\left[-\Delta \alpha\left(\cos ^{2} \theta\right)_{k l}-\alpha^{\perp} \delta_{k l}\right]|\overrightarrow{\mathbb{E}}|^{2}
\end{aligned}
$$

Because the rotational eigenstates $|k\rangle=|J, M\rangle=Y_{J M}(\theta, \phi)$ are spherical harmonics,

$$
\left(\cos ^{2} \theta\right)_{k l}=\left\langle J, M\left|\cos ^{2} \theta\right| J^{\prime}, M^{\prime}\right\rangle
$$

is nonvanishing only for $M=M^{\prime}$ and $J=J^{\prime}, J^{\prime} \pm 2$. Therefore, we obtain

$$
\rho_{J+2, J, M}^{(1)}=\frac{i}{2 \hbar}\left(\rho_{J, M}^{(0)}-\rho_{J+2, M}^{(0)}\right) \Delta \alpha\left(\cos ^{2} \theta\right)_{J+2, J}^{M}\left[\mathrm{e}^{\left(-\gamma_{J+2, J}-i \omega_{J+2, J}\right) t} *|\overrightarrow{\mathbb{E}}|^{2}\right]
$$

Note that $\rho_{J, J+2, M}^{(1)}$ is implicitly considered as well since

$$
\rho_{J, J+2, M}^{(1)}=\left(\rho_{J+2, J, M}^{(1)}\right)^{*} \text { and }\left[\left(\cos ^{2} \theta\right)_{J, J+2}^{M}\right]^{*}=\left(\cos ^{2} \theta\right)_{J+2, J}^{M} .
$$

The integration range in the convolution $\int_{-\infty}^{\infty}$ can be transformed into $\int_{-\infty}^{t}$ by assuming its integrand is defined only within the range of $[0, t]$ which is true for pulses. 
Eq. (18) is then incorporated into Eq. (11) and becomes

$$
\begin{aligned}
\left\langle\cos ^{2} \theta\right\rangle_{t} & =\operatorname{Tr}\left[\hat{\rho} \cos ^{2} \theta\right] \\
& =\sum_{k l}\left(\rho_{k l}^{(0)}+\rho_{k l}^{(1)}\right)\left(\cos ^{2} \theta\right)_{l k} \\
& =\sum_{k} \rho_{k}^{(0)}\left(\cos ^{2} \theta\right)_{k k}+\sum_{k l} \rho_{k l}^{(1)}\left(\cos ^{2} \theta\right)_{l k} \quad \because \rho_{k l}^{(0)}=\rho_{k}^{(0)} \delta_{k l} \\
& =\frac{1}{3}+\sum_{J M}\left(\rho_{J+2, J, M}^{(1)}+\rho_{J, J+2, M}^{(1)}\right)\left(\cos ^{2} \theta\right)_{J+2, J}^{M} \quad \because\left(\cos ^{2} \theta\right)_{J+2, J}^{M} \text { is real } \\
& =\frac{1}{3}+\sum_{J M} 2 \operatorname{Re}\left[\rho_{J+2, J, M}^{(1)}\right]\left(\cos ^{2} \theta\right)_{J+2, J}^{M}
\end{aligned}
$$

We then obtain

$$
\begin{aligned}
\epsilon & =\epsilon(t \rightarrow-\infty)+\sum_{J M} 2 N_{g} \Delta \alpha \operatorname{Re}\left[\rho_{J+2, J, M}^{(1)}\right]\left[\left(\cos ^{2} \theta\right)_{J+2, J}^{M}\right]^{2} \\
& =\epsilon(t \rightarrow-\infty)-\sum_{J M} \frac{1}{\hbar} N_{g}(\Delta \alpha)^{2}\left(\rho_{J, M}^{(0)}-\rho_{J+2, M}^{(0)}\right)\left[\left(\cos ^{2} \theta\right)_{J+2, J}^{M}\right]^{2} \operatorname{Im}\left[\mathrm{e}^{\left(-\gamma_{J+2, J}-i \omega_{J+2, J}\right) t} *|\overrightarrow{\mathbb{E}}|^{2}\right] \\
& =\epsilon(t \rightarrow-\infty)+\sum_{J M} \frac{1}{\hbar} N_{g}(\Delta \alpha)^{2}\left(\rho_{J, M}^{(0)}-\rho_{J+2, M}^{(0)}\right)\left[\left(\cos ^{2} \theta\right)_{J+2, J}^{M}\right]^{2} \operatorname{Im}\left[\mathrm{e}^{\left(-\gamma_{\left.J+2, J+i \omega_{J+2, J}\right) t} *|\overrightarrow{\mathbb{E}}|^{2}\right]}\right.
\end{aligned}
$$

By applying [34]

$$
\begin{aligned}
\sum_{M=-J}^{J}\left[\left(\cos ^{2} \theta\right)_{J+2, J}^{M}\right]^{2} & =\sum_{M=-J}^{J} \frac{\left[(J+2)^{2}-M^{2}\right]\left[(J+1)^{2}-M^{2}\right]}{(2 J+1)(2 J+3)^{2}(2 J+5)} \\
& =\frac{2}{15} \frac{(J+2)(J+1)}{2 J+3},
\end{aligned}
$$

Eq. (20) becomes

$\epsilon=\epsilon(t \rightarrow-\infty)+\sum_{J} \frac{2}{15 \hbar} N_{g}(\Delta \alpha)^{2}\left(\rho_{J}^{(0)}-\rho_{J+2}^{(0)}\right) \frac{(J+2)(J+1)}{2 J+3} \operatorname{Im}\left[\mathrm{e}^{\left(-\gamma_{J+2, J}+i \omega_{J+2, J}\right) t} *|\overrightarrow{\mathbb{E}}|^{2}\right]$,

where $\hat{\rho}^{(0)}$ is independent of $M: \rho_{J}^{(0)}=\rho_{J, M}^{(0)}$.

If we ignore the highly-oscillatory term in $|\overrightarrow{\mathbb{E}}|^{2}$, we can approximate $|\overrightarrow{\mathbb{E}}|^{2}$ as $|\overrightarrow{\mathcal{E}}|^{2} / 2$.

Because $\overrightarrow{\mathbb{P}}=\Delta \epsilon \overrightarrow{\mathbb{E}}=\left[R(t) *|\overrightarrow{\mathcal{E}}|^{2}\right] \overrightarrow{\mathbb{E}}$, we can then obtain the Raman response function,

$$
\begin{aligned}
R^{\mathrm{rot}} & =N_{g} \sum_{J} \frac{1}{15 \hbar}(\Delta \alpha)_{J}^{2}\left(\rho_{J}^{(0)}-\rho_{J+2}^{(0)}\right) \frac{(J+2)(J+1)}{2 J+3} \mathrm{e}^{-\gamma_{2}^{0 J \rightarrow 0 J+2} t} \sin \left(\omega_{0 J+2,0 J} t\right) \\
& =N_{g} \frac{1}{15 \hbar}(\Delta \alpha)^{2} \mathrm{e}^{-\gamma_{2}^{\mathrm{rot}} t} \sum_{J}\left(\rho_{J}^{(0)}-\rho_{J+2}^{(0)}\right) \frac{(J+2)(J+1)}{2 J+3} \sin \left(\omega_{0 J+2,0 J} t\right),
\end{aligned}
$$

where all dephasing times $T_{2}^{\text {rot }}=\frac{1}{\gamma_{2}^{\text {rot }}}=\frac{1}{\gamma_{2}^{0 J} \rightarrow 0 J+2}$ are assumed to be the same. 


\subsection{Vibrational Raman}

Although Wahlstrand et al. provide a detailed derivation [35], we derive the equation with a different approach that starts from the Maxwell-Bloch equations [7, 40,41].

$$
\begin{aligned}
\dot{w} & =-\gamma_{1}(w+1)-\frac{2[\alpha]_{a b}}{\hbar} \operatorname{Im}\left\{\rho_{a b}\right\}|\overrightarrow{\mathbb{E}}(t)|^{2} \\
\dot{\rho}_{a b} & =\left(-\gamma_{2}+i \omega_{b a}\right) \rho_{a b}+\frac{i}{2 \hbar}\left[\left([\alpha]_{a a}-[\alpha]_{b b}\right) \rho_{a b}+[\alpha]_{a b} w\right]|\overrightarrow{\mathbb{E}}(t)|^{2},
\end{aligned}
$$

where $[\rho]$ is the density matrix so that $w=\rho_{b b}-\rho_{a a}$ is the population inversion, $\gamma_{1}$ and $\gamma_{2}$ are the dephasing time of the coherence and the decay time of the upper-state population, respectively, $[\alpha]$ is the molecular polarizability matrix.

We assume that the applied field only perturbs the system such that

$$
\begin{aligned}
& \rho_{b b} \approx 0 \quad \Rightarrow \quad w \approx-1 \\
& \rho_{a b} \approx 0 .
\end{aligned}
$$

Eq. (24b) then can be solved by use of an integrating factor:

$$
\rho_{a b}(t)=-\frac{i}{2 \hbar}[\alpha]_{a b}\left[\mathrm{e}^{\left(-\gamma_{2}+i \omega_{b a}\right) t} *|\overrightarrow{\mathbb{E}}(t)|^{2}\right]
$$

The Raman polarization

$$
\begin{aligned}
\overrightarrow{\mathbb{P}}_{R} & =N_{g}\langle\hat{\alpha} \overrightarrow{\mathbb{E}}\rangle=N_{g}(\hat{\rho} \hat{\alpha}) \overrightarrow{\mathbb{E}} \\
& =N_{g}\left[[\alpha]_{a a}+\left([\alpha]_{b b}-[\alpha]_{a a}\right) \rho_{b b}+2[\alpha]_{a b} \operatorname{Re}\left\{\rho_{a b}\right\}\right] \overrightarrow{\mathbb{E}},
\end{aligned}
$$

in which $N_{g}[\alpha]_{a a}$ is the linear term and is taken into account in the propagation constant $\beta(\omega)$. Hence, the nonlinear Raman polarization is

$$
\begin{aligned}
\overrightarrow{\mathbb{P}}_{R, \text { nonlinear }} & =2 N_{g}[\alpha]_{a b} \operatorname{Re}\left\{\rho_{a b}\right\} \overrightarrow{\mathbb{E}} \\
& =\frac{1}{\hbar} N_{g}[\alpha]_{a b}^{2}\left[\mathrm{e}^{-\gamma_{2} t} \sin \left(\omega_{b a} t\right) *|\overrightarrow{\mathbb{E}}(t)|^{2}\right]
\end{aligned}
$$

By comparing the nonlinear Raman polarization in [39] (along with careful treatments of analytic signals) with Eq. (28), we derive the following relation

$$
[\alpha]_{a b}=\sqrt{\frac{\hbar}{2 \mu \omega_{b a}}}\left(\frac{\mathrm{d} \alpha}{\mathrm{d} \mathbb{Q}}\right)_{0}
$$

where $\mu$ is the reduced mass of the gas molecule, $\mathbb{Q}$ is its normal coordinate, and the subscript 0 denotes that the derivative is taken at equilibrium. With it, we obtain

$$
\overrightarrow{\mathbb{P}}_{R, \text { nonlinear }}=\frac{1}{2 \mu \omega_{b a}} N_{g}\left(\frac{\mathrm{d} \alpha}{\mathrm{d} \mathbb{Q}}\right)_{0}^{2}\left[\mathrm{e}^{-\gamma_{2} t} \sin \left(\omega_{b a} t\right) *|\overrightarrow{\mathbb{E}}(t)|^{2}\right]
$$

Next we consider the population of each state and add one more term corresponding to different rotational energy states [35]. Eq. (30) becomes

$$
\begin{array}{r}
\overrightarrow{\mathbb{P}}_{R, \text { nonlinear }=} \sum_{J} N_{g} \frac{(2 J+1) \rho_{J}^{(0)}}{2 \mu \omega_{1 J, 0 J}}\left[\left(\frac{\mathrm{d} \alpha}{\mathrm{d} \mathbb{Q}}\right)_{0}^{2}+\frac{4}{45} \frac{J(J+1)}{(2 J-1)(2 J+3)}\left(\frac{\mathrm{d}(\Delta \alpha)}{\mathrm{d} \mathbb{Q}}\right)_{0}^{2}\right] \times \\
{\left[\mathrm{e}^{-\gamma_{2} t} \sin \left(\omega_{1 J, 0 J} t\right) *|\overrightarrow{\mathbb{E}}(t)|^{2}\right] \overrightarrow{\mathbb{E}}}
\end{array}
$$


With $|\overrightarrow{\mathbb{E}}|^{2} \approx|\overrightarrow{\mathcal{E}}|^{2} / 2$ and $\overrightarrow{\mathbb{P}}=\left[R(t) *|\overrightarrow{\mathcal{E}}|^{2}\right] \overrightarrow{\mathbb{E}}$, we have the vibrational Raman response for the $\mathrm{Q}$ branch,

$$
R^{\mathrm{vib}}=N_{g} \frac{1}{4 \mu} \mathrm{e}^{-\gamma_{2}^{\mathrm{vib}} t} \sum_{J}(2 J+1) \rho_{J}^{(0)} \frac{\left(\frac{\mathrm{d} \alpha}{\mathrm{dQ}}\right)_{0}^{2}+\frac{4}{45} \frac{J(J+1)}{(2 J-1)(2 J+3)}\left(\frac{\mathrm{d}(\Delta \alpha)}{\mathrm{d} \mathbb{Q}}\right)_{0}^{2}}{\omega_{1 J, 0 J}} \sin \left(\omega_{1 J, 0 J} t\right)
$$

\subsection{Required steps in numerical simulations}

Due to long dephasing times of the Raman responses, the operation of the convolution (*) can't be performed directly with the circular convolution theorem unless the time window in simulations is large enough to cover the entire Raman response which is typically on the order of 1 to $10 \mathrm{~ns}$. This is undesirable especially if the physical phenomenon of interest happens on the time scale of less than a few picoseconds. Therefore, in each nonlinear step of ERK4(3)-IP [42], doubling the time window by filling one side with dummy zeros is required to apply circular convolution theorem and avoids aliasing numerically. Only the signal in the original time window contains useful information and is recovered back after the nonlinear operation.

Because discrete Raman transitions generate signals far from the pump frequency, aliasing occurs if the frequency window isn't large enough. Due to various orders of Raman transitions, aliasing is difficult to avoid no matter how large the frequency window is. Hence, in each nonlinear step, we extend the frequency window by three times by filling both edges with zeros. After the computation of the Raman response, we downsample the frequency window back to its original size and discard the high and low frequency parts. This operation avoids the potential aliasing resulting from Raman transitions of various orders and guarantees that the computation considers only the physics within the desired frequency window.

\subsection{Parameter values}

In tables 1 to 6 , the parameter values for both $\mathrm{H}_{2}$ and $\mathrm{N}_{2}$ are listed. $\mathrm{N}_{2}$ has been commonly used in hollow-core fibers. It will be used as an example to explain why $\mathrm{H}_{2}$ is advantageous for efficient Raman-soliton formation.

Table 1. Refractive index $n_{0}$ at $0{ }^{\circ} \mathrm{C}$ and 1 bar. At temperature $T, n=1+\left(n_{0}-1\right) \rho$, where $\rho$ is the gas density in amagats. $\lambda$ is in $\mu \mathrm{m}$.

\begin{tabular}{c|l}
\hline Molecule & $n_{0}$ \\
\hline $\mathrm{H}_{2}$ & $1+\frac{0.0148956}{180.7-\lambda^{-2}}+\frac{0.0049037}{92-\lambda^{-2}}[43]$ \\
$\mathrm{N}_{2}$ & $1+6.8552 \times 10^{-5}+\frac{3.243157 \times 10^{-2}}{144-\lambda^{-2}}[44]$ \\
\hline
\end{tabular}

Table 2. Nonlinear refractive index $n_{2}=n_{2}^{0} \rho$, where $\rho$ is the gas density in amagats. For $\mathbf{N}_{2}, n_{2}^{0}=10^{24} \frac{P_{n_{2}}^{-1}}{\lambda_{0}^{-2}-\lambda^{-2}}\left(10^{-24} \mathrm{~m}^{2} / \mathrm{W} /\right.$ bar $)[\mathbf{4 5}]$.

\begin{tabular}{c|ccc}
\hline Molecule & $n_{2}^{0}\left(10^{-24} \mathrm{~m}^{2} / \mathrm{W} /\right.$ bar $)$ & $P_{n_{2}}(\mathrm{~W})$ & $\lambda_{0}(\mathrm{~m})$ \\
\hline $\mathrm{H}_{2}$ & $6.5[35,46]$ & & \\
$\mathrm{N}_{2}$ & & $14.63 \times 10^{9}$ & $0.3334 \times 10^{-6}$ \\
\hline
\end{tabular}


Table 3. Nuclear spin statistics $g_{J}[47]$

\begin{tabular}{c|cc}
\hline Molecule & $J$ is even & $J$ is odd \\
\hline $\mathrm{H}_{2}$ & 1 & 3 \\
$\mathrm{~N}_{2}$ & 6 & 3 \\
\hline
\end{tabular}

Table 4. Constants of energy states [48-50]

\begin{tabular}{c|ccc}
\hline Molecule & $B_{e}\left(\mathrm{~cm}^{-1}\right)$ & $D_{e}\left(\mathrm{~cm}^{-1}\right)$ & $\omega_{\text {vib }}\left(\mathrm{cm}^{-1}\right)$ \\
\hline $\mathrm{H}_{2}$ & 60.8 & $1.6 \times 10^{-2}$ & $4155[6]$ \\
$\mathrm{N}_{2}$ & 1.98958 & $5.76 \times 10^{-6}$ & 2329.9 \\
\hline
\end{tabular}

Table 5. Polarizability. a.u. $=1.64878 \times 10^{-41} \mathrm{Fm}^{2} \cdot \mu$ is the reduced mass of the molecule. Their values are carefully adjusted to obtain agreements with other studies $[14,34,51-53]$.

\begin{tabular}{c|ccc}
\hline Molecule & $\Delta \alpha$ (a.u.) & $\alpha^{\prime} / \sqrt{\mu}(\mathrm{Fm} / \sqrt{\mathrm{kg}})$ & $(\Delta \alpha)^{\prime} / \sqrt{\mu}(\mathrm{Fm} / \sqrt{\mathrm{kg}})$ \\
\hline $\mathrm{H}_{2}$ & $2.23[54,55]$ & $3.88 \times 10^{-17}[35]$ & $2.82 \times 10^{-17}[55]$ \\
$\mathrm{N}_{2}$ & $4.54[36,54]$ & $1.80 \times 10^{-17}[50]$ & $2.29 \times 10^{-17}[50]$ \\
\hline
\end{tabular}

Table 6. Dephasing time $T_{2}=\frac{1}{\pi \Delta v}=\frac{1}{\gamma_{2}} \cdot \rho$ is the gas density in amagats and $T$ is the temperature in $\mathrm{K}$. Below $\Delta v$ is under $\mathrm{MHz}$.

\begin{tabular}{c|cc}
\hline Molecule & vibrational Raman $\Delta v^{\text {vib }}$ & rotational Raman $\Delta v^{\text {rot }}$ \\
\hline $\mathrm{H}_{2}$ & $\frac{309}{\rho}\left[\frac{T}{298}\right]^{0.92}+\left[51.8+0.152(T-298)+4.85 \times 10^{-4}(T-298)^{2}\right] \rho[56]$ & $\frac{6.15}{\rho}+114 \rho[6]$ \\
$\mathrm{N}_{2}$ & $22.5, \rho<10[6]$ & $3570 \rho[6]$ \\
\hline
\end{tabular}

\section{Simulations of pulse propagation in nitrogen-filled hollow-core fiber}

To demonstrate what will happen if there are potentially many Raman transitions, we consider nitrogen-filled hollow-core fiber. Because $\mathrm{N}_{2}$ has closely-spaced rotational energy states, the pulse is affected by multiple Raman transitions simultaneously. To illuminate the underlying physics and compare $\mathrm{H}_{2}$ and $\mathrm{N}_{2}$, we simulated injection of a 20 -fs fundamental soliton into a 30- $\mu$ m-core-diameter AR-HCF with 10 bar of $\mathrm{H}_{2}$ or $\mathrm{N}_{2}$. Under this gas pressure, the dispersion is dominated by the anomalous waveguide dispersion. The fundamental soliton energies are $220 \mathrm{~nJ}$ for $\mathrm{H}_{2}$ and $180 \mathrm{~nJ}$ for $\mathrm{N}_{2}$. A pulse duration of $20 \mathrm{fs}$ is chosen to obtain a strong fundamental soliton and broad bandwidth for clear demonstration of SSFS.

Figs. 6(a-c) show the SSFS in $\mathrm{H}_{2}$ while Figs. 6(d-f) show it in $\mathrm{N}_{2}$. In contrast to the single clean sech-like Raman soliton in $\mathrm{H}_{2}$, there is a wide spectrum in $\mathrm{N}_{2}$ [Fig. 6(b) and 6(e)]. In Fig. 6(d), at 1-m and 2-m propagation distances, the Raman soliton develops blue spectral peaks. This results from the interplay of various Raman transitions in $\mathrm{N}_{2}$. If we filter out only the reddest 
spectral peak, we can see, in Fig. 6(c), all the energy contributes to the 45-fs Raman soliton. However, in Fig. 6(f), the pulse develops a long pesdestal while the filtered pulse takes up only a fraction of the energy and has 80-fs pulse duration. To demonstrate the difference between the Raman transitions in both gases, we define the strength of the transition from the rotational energy state $J$ to $J+2$ as the $R_{J}^{\text {coeff }}$ in Eq. (33), which is derived from Eq. (23). In Fig. $6(\mathrm{~g}$ ), we can see that there is only one dominant Raman transition in $\mathrm{H}_{2}$ around $18 \mathrm{THz}$ which results from $\mathrm{S}(1)$. On the other hand, there is a cluster of transitions with similar strengths at $3 \mathrm{THz}$ in $\mathrm{N}_{2}$.

$$
R^{\mathrm{rot}}=\mathrm{e}^{-\gamma_{2}^{\mathrm{rot}} t} \sum_{J} R_{J}^{\text {coeff }} \sin \left(\omega_{0 J+2,0 J} t\right)
$$

(a)

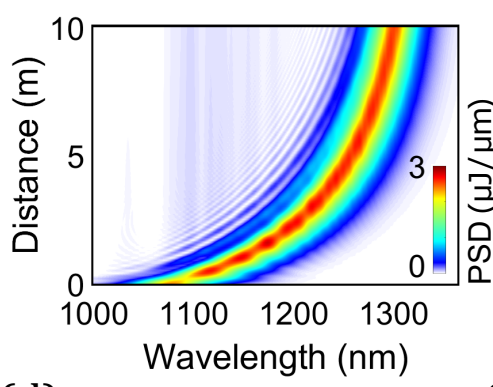

(d)

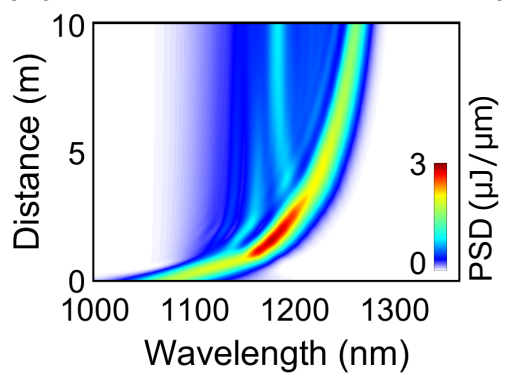

(b)

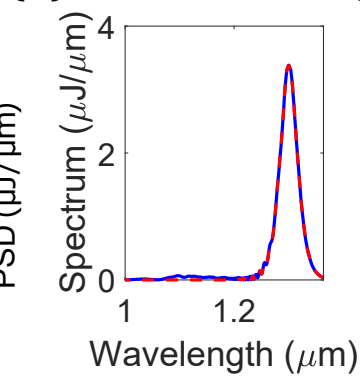

(e)

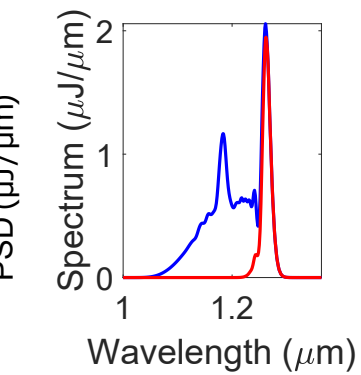

(c)

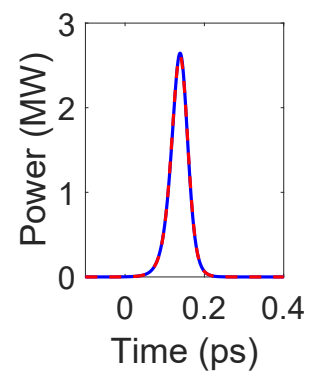

(f)

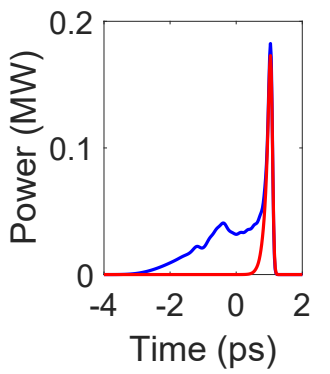

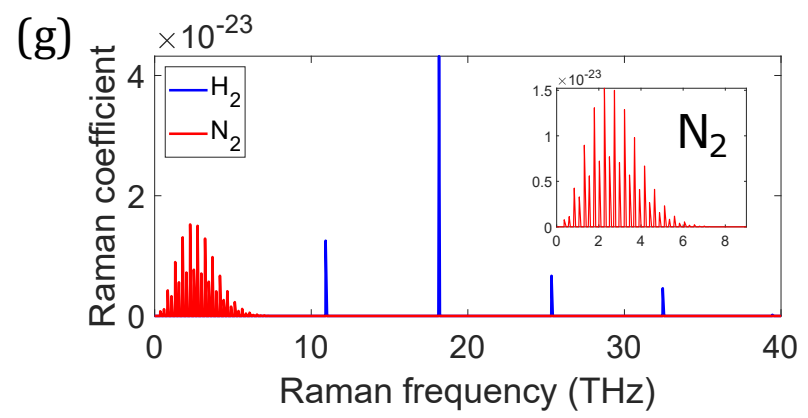

Fig. 6. Simulations with a fundamental soliton. Simulated (a) spectral evolution, (b) output spectrum, and (c) output pulse before (blue) and after (red) filtering out the reddest spectral peak in $\mathrm{H}_{2}$. Red dashed lines are used to clearly show their overlap. Simulated (d) spectral evolution, (e) output spectrum, and (f) output pulse before (blue) and after (red) filtering out the reddest spectral peak in $\mathrm{N}_{2}$. (g) The strength of each Raman transition. It's calculated under the SI unit with $A(z, t)$ having the unit of $\sqrt{\mathrm{W}}$. The inset in $(\mathrm{g})$ is a close-view of the $\mathrm{N}_{2}$ Raman strength. 


\section{Measurements of the output spatial profiles}

Fig. 7 shows the measured output spatial profiles of the filtered Raman beam (Fig. 2) at pressures from 0 bar to 80 bar. In Fig. 8, the spatial profile at 40 bar is fitted to a Bessel function of the first kind as an example to show that these profiles correspond to the fundamental transverse mode of the AR-HCF.
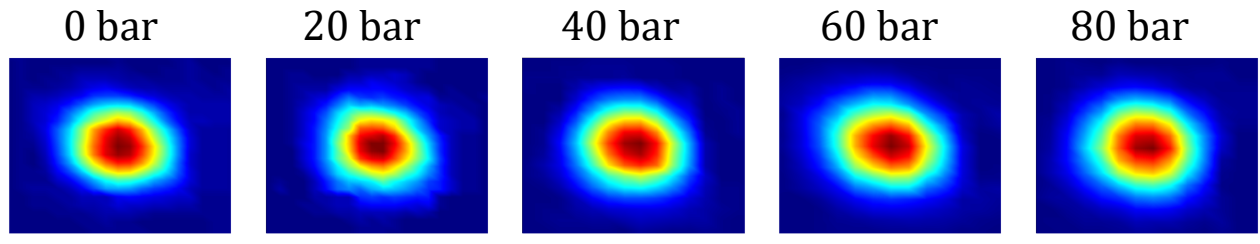

Fig. 7. Measured output spatial profiles with $\mathrm{H}_{2}$ pressure from 0 bar to 80 bar.

(a)

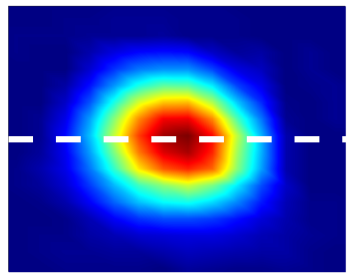

(b)

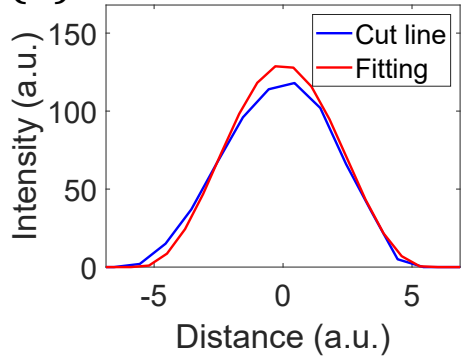

Fig. 8. The (white) cutline of the spatial profile at 40-bar $\mathrm{H}_{2}$ pressure (a) is shown in (b). The central part is fitted to $c \cdot J_{0}\left(k\left(x-x_{0}\right)\right)$, the fundamental transverse mode of a hollow-core fiber.

\section{Resonance-induced dispersive-wave generation}

Due to the sharp dispersion slope near a resonance, efficient narrowband dispersive-wave generation occurs at high gas pressures despite the high confinement loss [31,57]. Fig. 9(a) depicts the phase-matching relation of a dispersive wave, which is calculated from

$$
\Delta \beta_{\mathrm{DW}}=\beta(\omega)-\left[\beta\left(\omega_{p}\right)+\beta_{1}\left(\omega-\omega_{p}\right)+\beta_{\mathrm{DW}}^{\mathrm{Kerr}}\right],
$$

where $\beta(\omega)$ is the propagation constant at angular frequency $\omega$ and those in the square brackets represent the propagation constant of the pump pulse. $\beta\left(\omega_{p}\right)$ and $\beta_{1}$ are the propagation constant and the inverse group velocity at the pump wavelength, $\omega_{p}$ is the pump angular frequency, $\beta_{\mathrm{DW}}^{\mathrm{Kerr}}=\gamma P_{0} \omega / \omega_{p}$ is the nonlinear contribution of the optical Kerr effect, $\gamma=\frac{n_{2} \omega_{p}}{c A_{\mathrm{eff}}}$ is the nonlinear coefficient, $P_{0}$ is the peak power of the pump pulse. Because of the S-shape of the dispersion curve near resonance, there is a maximum of three phase-matched dispersivewave wavelengths where $\Delta \beta_{\text {DW }}$ approaches zero. At $1030 \mathrm{~nm}$, there are three curves, (1-3) in Fig. 9(a), where dispersive waves are phase-matched with the pump pulse. With increasing pump wavelength, only one phase-matching curve is observed. Since our gain-managed pump pulse has a broadband spectrum from 1000 to $1100 \mathrm{~nm}$, multiple dispersive waves are generated. In Fig. 9(c), we experimentally observed multiple dispersive waves. The first dispersive wave is generated and red-shifts from 540 to $570 \mathrm{~nm}$ as the gas pressure increases. The second dispersive 
wave, however, stays around $665 \mathrm{~nm}$ with different pressures. Finally, the third one red-shifts as the first one but is rather broadband. These features are consistent with the calculation of the phase-matching relation in Fig. 9(a).

Not only dispersive waves but also four-wave mixing (FWM) becomes phase-matched near a resonance. The FWM phase-matching relation is

$$
\triangle \beta_{\mathrm{FWM}}=\beta\left(\omega_{s}\right)+\beta\left(\omega_{i}\right)-2 \beta\left(\omega_{p}\right)+\beta_{\mathrm{FWM}}^{\mathrm{Kerr}},
$$

where $\omega_{s}$ and $\omega_{i}$ are angular frequencies of the signal and the idler that satisfy $\omega_{s}+\omega_{i}-2 \omega_{p}=0$. $\beta_{\mathrm{FWM}}^{\mathrm{Kerr}}=2 \gamma P_{0}$ is the nonlinear contribution of the optical Kerr effect. Fig. 9(b) shows the phase-matching relation when the pump wavelength is $1080 \mathrm{~nm}$. The two white curves are the phase-matched signal and the idler. They stay around the pump wavelength and don't contribute to the visible wavelengths. The same feature is found with different pump wavelengths, so they are not shown here.

(a)

Pump wavelength

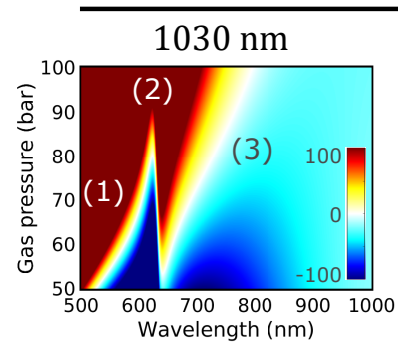

(b)
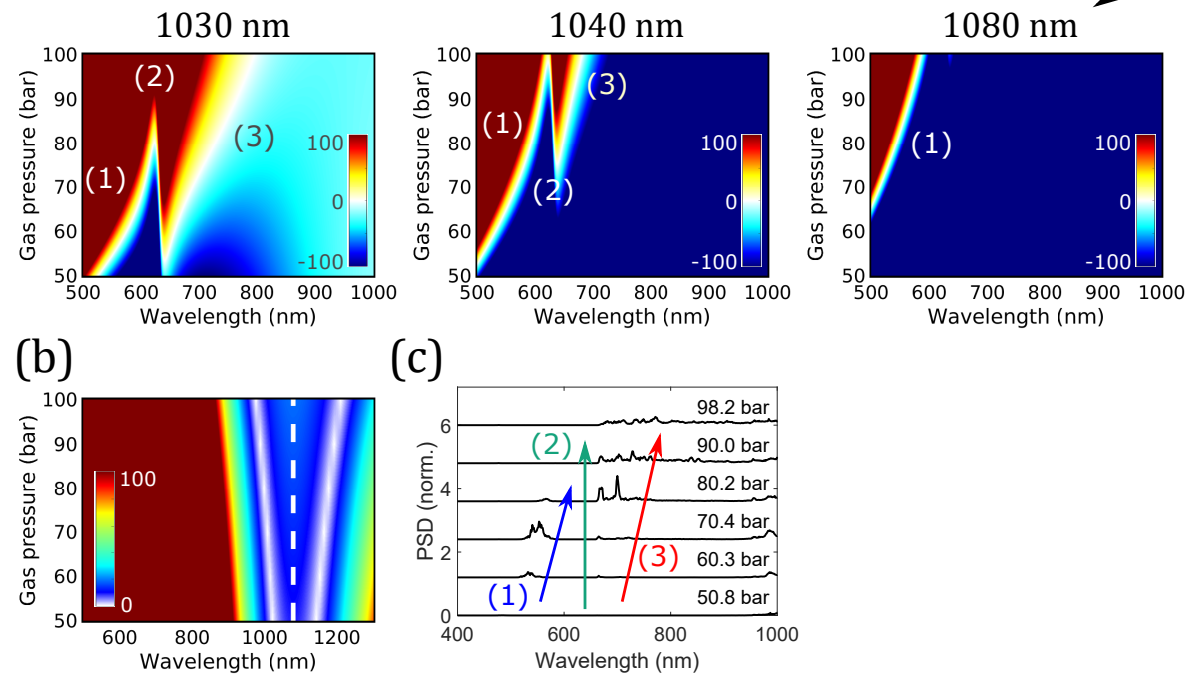

(c)

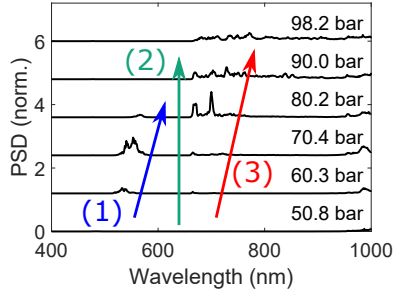

Fig. 9. (a) The evolution of the phase-matching relation ( $\triangle \beta_{\mathrm{DW}}$ ) of the dispersive wave at different pump wavelengths. (b) The phase-matching relation $\left(\left|\Delta \beta_{\mathrm{FWM}}\right|\right)$ of the four-wave mixing. The white dashed line represents the pump wavelength. (c) The close-view of the visible spectra of Fig. 2(a) in the manuscript. (1-3) are three efficient dispersive-wave emissions where $\Delta \beta_{\mathrm{DW}} \sim 0$. The units of both $\Delta \beta$ are $1 / \mathrm{m}$.

\section{Discussion of photoionization in scaling of SSFS to higher pulse energy}

To generate a Raman soliton in $\mathrm{H}_{2}$ through SSFS with high efficiency, a short pulse is preferred. However, to scale to energies of several microjoules, the peak power of the input pulse can be high enough to ionize $\mathrm{H}_{2}$. This may lead to phenomena such as photoionization-induced blue-shift [2,58]. Keldysh theory provides a framework for quantitative analysis of this issue [59]. In particular, the Keldysh parameter $\gamma$ can be used to delineate the transition between the high-intensity tunneling domain $(\gamma \ll 1)$ and the low-intensity multiphoton ionization $(\gamma \gg 1)$. For the $2-\mu \mathrm{J}$ pulses considered in the manuscript, the Keldysh parameter is 1.7 , which is similar to the case in [7], where ionization does not have a significant effect. $\gamma \approx 1$ for $5.8-\mu \mathrm{J}$ pulses in a hydrogen-filled fiber of a $30-\mu \mathrm{m}$ core diameter; in that case, photoionization will be 
appreciable $[2,58,60]$. To avoid ionization, Jenkins et al. have proposed use of the divided-pulse technique [61] to reduce the peak power [62]. This technique has also been applied experimentally in SSFS but with a solid-core photonic crystal fiber [63]. Divided-pulse SSFS may be a way to avoid photoionization in scaling SSFS in hydrogen to energies above several microjoules. 


\section{References}

1. N. Y. Joly, J. Nold, W. Chang, P. Hölzer, A. Nazarkin, G. K. L. Wong, F. Biancalana, and P. St. J. Russell, "Bright Spatially Coherent Wavelength-Tunable Deep-UV Laser Source Using an Ar-Filled Photonic Crystal Fiber," Phys. Rev. Lett. 106, 203901 (2011).

2. M. F. Saleh, W. Chang, P. Hölzer, A. Nazarkin, J. C. Travers, N. Y. Joly, P. St. J. Russell, and F. Biancalana, "Theory of Photoionization-Induced Blueshift of Ultrashort Solitons in Gas-Filled Hollow-Core Photonic Crystal Fibers," Phys. Rev. Lett. 107, 203902 (2011).

3. A. I. Adamu, M. S. Habib, C. R. Petersen, J. E. A. Lopez, B. Zhou, A. Schülzgen, M. Bache, R. Amezcua-Correa, O. Bang, and C. Markos, "Deep-UV to Mid-IR Supercontinuum Generation driven by Mid-IR Ultrashort Pulses in a Gas-filled Hollow-core Fiber," Sci. Rep. 9, 4446 (2019).

4. J. C. Travers, T. F. Grigorova, C. Brahms, and F. Belli, "High-energy pulse self-compression and ultraviolet generation through soliton dynamics in hollow capillary fibres," Nat. Photon. 13, 547-554 (2019).

5. U. Elu, L. Maidment, L. Vamos, F. Tani, D. Novoa, M. H. Frosz, V. Badikov, D. Badikov, V. Petrov, P. St. J. Russell, and J. Biegert, "Seven-octave high-brightness and carrier-envelope-phase-stable light source," Nat. Photon. 15, 277-280 (2021)

6. M. J. Weber, CRC Handbook of Laser Science and Technology Supplement 2 (CRC Press, 1994).

7. F. Belli, A. Abdolvand, W. Chang, J. C. Travers, and P. St. J. Russell, "Vacuum-ultraviolet to infrared supercontinuum in hydrogen-filled photonic crystal fiber," Optica 2, 292-300 (2015).

8. M. F. Saleh, A. Armaroli, T. X. Tran, A. Marini, F. Belli, A. Abdolvand, and F. Biancalana, "Raman-induced temporal condensed matter physics in gas-filled photonic crystal fibers," Opt. Express 23, 11879-11886 (2015).

9. S. Loranger, P. St. J. Russell, and D. Novoa, "Sub-40 fs pulses at $1.8 \mu \mathrm{m}$ and MHz repetition rates by chirp-assisted Raman scattering in hydrogen-filled hollow-core fiber," J. Opt. Soc. Am. B 37, 3550-3556 (2020).

10. D. G. Ouzounov, F. R. Ahmad, D. Müller, N. Venkataraman, M. T. Gallagher, M. G. Thomas, J. Silcox, K. W. Koch, and A. L. Gaeta, "Generation of Megawatt Optical Solitons in Hollow-Core Photonic Band-Gap Fibers," Science 301, 1702 (2003).

11. F. Luan, J. C. Knight, P. St. J. Russell, S. Campbell, D. Xiao, D. T. Reid, B. J. Mangan, D. P. Williams, and P. J. Roberts, "Femtosecond soliton pulse delivery at $800 \mathrm{~nm}$ wavelength in hollow-core photonic bandgap fibers," Opt. Express 12, 835-840 (2004).

12. F. Gérôme, P. Dupriez, J. Clowes, J. C. Knight, and W. J. Wadsworth, "High power tunable femtosecond soliton source using hollow-core photonic bandgap fiber, and its use for frequency doubling," Opt. Express 16, 2381-2386 (2008).

13. P. A. Carpeggiani, G. Coccia, G. Fan, E. Kaksis, A. Pugžlys, A. Baltuška, R. Piccoli, Y.-G. Jeong, A. Rovere, R. Morandotti, L. Razzari, B. E. Schmidt, A. A. Voronin, and A. M. Zheltikov, "Extreme Raman red shift: ultrafast multimode nonlinear space-time dynamics, pulse compression, and broadly tunable frequency conversion," Optica 7, 1349-1354 (2020).

14. J. E. Beetar, M. Nrisimhamurty, T.-C. Truong, G. C. Nagar, Y. Liu, J. Nesper, O. Suarez, F. Rivas, Y. Wu, B. Shim, and M. Chini, "Multioctave supercontinuum generation and frequency conversion based on rotational nonlinearity," Sci. Adv. 6, eabb5375 (2020).

15. R. Safaei, G. Fan, O. Kwon, K. Légaré, P. Lassonde, B. E. Schmidt, H. Ibrahim, and F. Légaré, "High-energy multidimensional solitary states in hollow-core fibres," Nat. Photon. 14, 733-739 (2020).

16. S. A. Dekker, A. C. Judge, R. Pant, I. Gris-Sánchez, J. C. Knight, C. M. de Sterke, and B. J. Eggleton, "Highly-efficient, octave spanning soliton self-frequency shift using a specialized photonic crystal fiber with low oh loss," Opt. Express 19, 17766-17773 (2011).

17. W. Bi, X. Li, Z. Xing, Q. Zhou, Y. Fang, W. Gao, L. Xiong, L. Hu, and M. Liao, "Wavelength conversion through soliton self-frequency shift in tellurite microstructured fiber with picosecond pump pulse," J. Appl. Phys. 119, 043102 (2016).

18. K. Wang and C. Xu, "Tunable high-energy soliton pulse generation from a large-mode-area fiber and its application to third harmonic generation microscopy," Appl. Phys. Lett. 99, 071112 (2011).

19. D. G. Ouzounov, T. Wang, M. Wang, D. D. Feng, N. G. Horton, J. C. Cruz-Hernández, Y.-T. Cheng, J. Reimer, A. S. Tolias, N. Nishimura, and C. Xu, "In vivo three-photon imaging of activity of GCaMP6-labeled neurons deep in intact mouse brain," Nat. Methods 14, 388-390 (2017).

20. D. M. Chow, D. Sinefeld, K. E. Kolkman, D. G. Ouzounov, N. Akbari, R. Tatarsky, A. Bass, C. Xu, and J. R. Fetcho, "Deep three-photon imaging of the brain in intact adult zebrafish," Nat. Methods 17, 605-608 (2020).

21. J. P. Gordon, "Theory of the soliton self-frequency shift," Opt. Lett. 11, 662-664 (1986).

22. F. M. Mitschke and L. F. Mollenauer, "Discovery of the soliton self-frequency shift," Opt. Lett. 11, 659-661 (1986).

23. M.-C. Chan, S.-H. Chia, T.-M. Liu, T.-H. Tsai, M.-C. Ho, A. A. Ivanov, A. M. Zheltikov, J.-Y. Liu, H.-L. Liu, and C.-K. Sun, "1.2- to 2.2- $\mu$ m Tunable Raman Soliton Source Based on a Cr:Forsterite Laser and a Photonic-Crystal Fiber," IEEE Photonics Technol. Lett. 20, 900-902 (2008).

24. H. Delahaye, C.-H. Hage, S. M. Bardet, I. Tiliouine, G. Granger, D. Gaponov, L. Lavoute, M. Jossent, S. Aleshkina, M. Bubnov, M. Salganskii, D. Lipatov, A. Guryanov, M. Likhachev, F. Louradour, and S. Février, "Generation of megawatt soliton at $1680 \mathrm{~nm}$ in very large mode area antiresonant fiber and application to three-photon microscopy," J. Opt. 23, 115504 (2021).

25. L. Rishøj, B. Tai, P. Kristensen, and S. Ramachandran, "Soliton self-mode conversion: revisiting Raman scattering 
of ultrashort pulses," Optica 6, 304-308 (2019).

26. G. Fan, R. Safaei, O. Kwon, V. Schuster, K. Légaré, P. Lassonde, A. Ehteshami, L. Arias, A. Laramée, J. BeaudoinBertrand, J. Limpert, Z. Tao, M. Spanner, B. E. Schmidt, H. Ibrahim, A. Baltuška, and F. Légaré, "High energy redshifted and enhanced spectral broadening by molecular alignment," Opt. Lett. 45, 3013-3016 (2020).

27. P. Sidorenko, W. Fu, and F. Wise, "Nonlinear ultrafast fiber amplifiers beyond the gain-narrowing limit," Optica 6, 1328-1333 (2019).

28. P. Sidorenko and F. Wise, "Generation of $1 \mu \mathrm{J}$ and 40 fs pulses from a large mode area gain-managed nonlinear amplifier," Opt. Lett. 45, 4084-4087 (2020).

29. M. S. Habib, J. E. Antonio-Lopez, C. Markos, A. Schülzgen, and R. Amezcua-Correa, "Single-mode, low loss hollow-core anti-resonant fiber designs," Opt. Express 27, 3824-3836 (2019).

30. M. Bache, M. S. Habib, C. Markos, and J. Lægsgaard, "Poor-man's model of hollow-core anti-resonant fibers," J. Opt. Soc. Am. B 36, 69-80 (2019).

31. F. Tani, F. Köttig, D. Novoa, R. Keding, and P. St. J. Russell, "Effect of anti-crossings with cladding resonances on ultrafast nonlinear dynamics in gas-filled photonic crystal fibers," Photon. Res. 6, 84-88 (2018).

32. F. Poletti and P. Horak, "Description of ultrashort pulse propagation in multimode optical fibers," J. Opt. Soc. Am. B 25, 1645-1654 (2008).

33. G. Agrawal, "Chapter 2 - pulse propagation in fibers," in Nonlinear Fiber Optics, (Academic Press, Boston, 2013), pp. 27-56, 5th ed.

34. Y.-H. Chen, S. Varma, A. York, and H. M. Milchberg, "Single-shot, space- and time-resolved measurement of rotational wavepacket revivals in $\mathrm{H}_{2}, \mathrm{D}_{2}, \mathrm{~N}_{2}, \mathrm{O}_{2}$, and $\mathrm{N}_{2} \mathrm{O}$," Opt. Express 15, 11341-11357 (2007).

35. J. K. Wahlstrand, S. Zahedpour, Y.-H. Cheng, J. P. Palastro, and H. M. Milchberg, "Absolute measurement of the ultrafast nonlinear electronic and rovibrational response in $\mathrm{H}_{2}$ and $\mathrm{D}_{2}$," Phys. Rev. A 92, 063828 (2015).

36. D. Langevin, J. M. Brown, M. B. Gaarde, and A. Couairon, "Determination of molecular contributions to the nonlinear refractive index of air for mid-infrared femtosecond laser-pulse excitation," Phys. Rev. A 99, 063418 (2019)

37. E. T. J. Nibbering, G. Grillon, M. A. Franco, B. S. Prade, and A. Mysyrowicz, "Determination of the inertial contribution to the nonlinear refractive index of air, $\mathrm{N}_{2}$, and $\mathrm{O}_{2}$ by use of unfocused high-intensity femtosecond laser pulses," J. Opt. Soc. Am. B 14, 650-660 (1997).

38. P. J. Bustard, B. J. Sussman, and I. A. Walmsley, "Phase-stable molecular phase modulation," (2008). H2 and N2 rotational dephasing time.

39. R. W. Boyd, "Chapter 3 - Quantum-Mechanical Theory of the Nonlinear Optical Susceptibility," in Nonlinear Optics, (Academic Press, Burlington, 2008), pp. 135-206, 3rd ed.

40. V. Kalosha and J. Herrmann, "Phase Relations, Quasicontinuous Spectra and Subfemtosecond Pulses in High-Order Stimulated Raman Scattering with Short-Pulse Excitation," Phys. Rev. Lett. 85, 1226-9 (2000).

41. F. L. Kien, K. Hakuta, and A. V. Sokolov, "Pulse compression by parametric beating with a prepared Raman coherence," Phys. Rev. A 66, 023813 (2002).

42. S. Balac and F. Mahé, "Embedded Runge-Kutta scheme for step-size control in the interaction picture method," Comput. Phys. Commun. 184, 1211-1219 (2013).

43. E. R. Peck and S. Huang, "Refractivity and dispersion of hydrogen in the visible and near infrared," J. Opt. Soc. Am. 67, 1550-1554 (1977).

44. E. R. Peck and B. N. Khanna, "Dispersion of Nitrogen,” J. Opt. Soc. Am. 56, 1059-1063 (1966).

45. J. M. Brown, A. Couairon, and M. B. Gaarde, "Ab initio calculations of the linear and nonlinear susceptibilities of $\mathrm{N}_{2}, \mathrm{O}_{2}$, and air in midinfrared laser pulses," Phys. Rev. A 97, 063421 (2018).

46. C. Köhler, R. Guichard, E. Lorin, S. Chelkowski, A. D. Bandrauk, L. Bergé, and S. Skupin, "Saturation of the nonlinear refractive index in atomic gases," Phys. Rev. A 87 (2013).

47. "Physical Chemistry II, 5.62 Lecture 13," MIT OpenCourseWare (2008).

48. D. Spelsberg and W. Meyer, "Static dipole polarizabilities of $\mathrm{N}_{2}, \mathrm{O}_{2}, \mathrm{~F}_{2}$, and $\mathrm{H}_{2} \mathrm{O}$," J. Chem. Phys. 101, 1282-1288 (1994).

49. W. Demtröder, "Diatomic Molecules," in Atoms, Molecules and Photons: An Introduction to Atomic-, Molecularand Quantum Physics, (Springer Berlin Heidelberg, Berlin, Heidelberg, 2010), pp. 327-381.

50. J. Lampel, U. Frieß, and U. Platt, "The impact of vibrational Raman scattering of air on DOAS measurements of atmospheric trace gases," Atmos Meas Tech 8, 3767-3787 (2015).

51. H. Li, W. Huang, Y. Cui, Z. Zhou, and Z. Wang, "Pure rotational stimulated Raman scattering in $\mathrm{H}_{2}$-filled hollow-core photonic crystal fibers," Opt. Express 28, 23881-23897 (2020).

52. A. V. Konyashchenko, L. L. Losev, V. S. Pazyuk, and S. Y. Tenyakov, "Frequency shifting of sub-100 fs laser pulses by stimulated Raman scattering in a capillary filled with pressurized gas," Appl. Phys. B 93, 455-461 (2008).

53. W. K. Bischel and M. J. Dyer, "Wavelength dependence of the absolute Raman gain coefficient for the Q(1) transition in $\mathrm{H}_{2}$, J. Opt. Soc. Am. B 3, 677-682 (1986).

54. N. J. Bridge, A. D. Buckingham, and J. W. Linnett, “The polarization of laser light scattered by gases," Proc. Royal Soc. London. Ser. A. Math. Phys. Sci. 295, 334-349 (1966).

55. W. Kolos and L. Wolniewicz, "Polarizability of the Hydrogen Molecule," J. Chem. Phys. 46, 1426-1432 (1967).

56. W. K. Bischel and M. J. Dyer, "Temperature dependence of the Raman linewidth and line shift for the Q(1) and $\mathrm{Q}(0)$ transitions in normal and para- $\mathrm{H}_{2}$," Phys. Rev. A 33, 3113-3123 (1986). Dephasing time for the $\mathrm{H} 2$ vibrational Raman. 
57. Y. Chen, Z. Huang, F. Yu, D. Wu, J. Fu, D. Wang, M. Pang, Y. Leng, and Z. Xu, "Photoionization-assisted, high-efficiency emission of a dispersive wave in gas-filled hollow-core photonic crystal fibers," Opt. Express 28 , 17076-17085 (2020).

58. M. F. Saleh and F. Biancalana, "Ultra-broadband supercontinuum generation in gas-filled photonic-crystal fibers: the epsilon-near-zero regime," Opt. Lett. 46, 1959-1962 (2021).

59. L. V. Keldysh, "Ionization in the Field of a Strong Electromagnetic Wave," J. Exp. Theor. Phys. 20, 1307-1314 (1965).

60. F. Köttig, D. Novoa, F. Tani, M. C. Günendi, M. Cassataro, J. C. Travers, and P. St. J. Russell, "Mid-infrared dispersive wave generation in gas-filled photonic crystal fibre by transient ionization-driven changes in dispersion," Nat. Commun. 8, 813 (2017).

61. S. Zhou, F. W. Wise, and D. G. Ouzounov, "Divided-pulse amplification of ultrashort pulses," Opt. Lett. 32, 871-873 (2007).

62. G. W. Jenkins, C. Feng, and J. Bromage, "Overcoming gas ionization limitations with divided-pulse nonlinear compression," Opt. Express 28, 31943-31953 (2020).

63. C. Zhang, V. Bucklew, P. Edwards, C. Janisch, and Z. Liu, "Divided pulse soliton self-frequency shift: a multi-color, dual-polarization, power-scalable, broadly tunable optical source,” Opt. Lett. 42, 502-505 (2017). 